\title{
Road to resilience. Articulación, ordenación y protección de la ribera urbana
}

\section{Ámbar Amelia Ceballos Peña}

Arquitecta, magíster en Urbanismo por la Universidad Politécnica de Cataluña, arq.ambaramelia@gmail.com.

\section{Francesc Peremiquel Lluch}

Doctor arquitecto; Departamento Urbanismo y Ordenación del Territorio. ETSAB. Universidad Politécnica de Cataluña, peremiquel@coac.net.

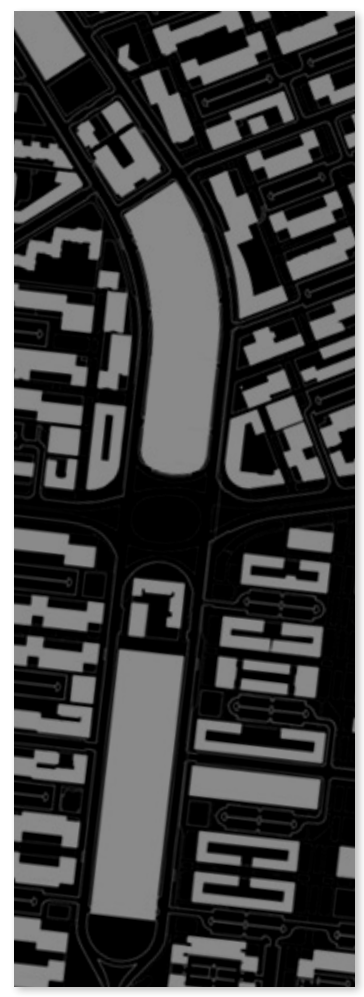




\title{
Resumen
}

La ciudad, como sistema de energía cambiante, debe adaptar sus mecanismos de defensa frente a impactos de naturaleza diversa en su proceso de transformación. La 'resiliencia', o capacidad para hacer frente a ellos, se ha convertido en uno de los principales objetivos que alcanzar en el correcto desarrollo de la ciudad contemporánea.

Siendo las crecidas de los ríos un impacto recurrente en ciudades fluviales, las riberas urbanas son un escenario oportuno para el estudio del comportamiento urbano-resiliente. "Road to Resilience" analiza, utilizando como caso de estudio el sistema hidrológico del río Ebro en su paso por la ciudad de Zaragoza, actuaciones que ejemplifican estrategias de anticipación, absorción o adaptación como respuesta ante las inundaciones mediante la articulación del sistema urbano y el fluvial, la ordenación de la ribera urbana y la implementación de formas de protección, desde una lógica de autodefensa.

\section{Palabras clave}

Resiliencia urbana; inundaciones; ribera urbana; Zaragoza.

\begin{abstract}
Road to resilience. Articulation, planning and protection of the urban riperian coast.

The city, as a changing energy system, must adapt its defense mechanisms against the diverse impacts in its process of transformation. 'Resilience', the ability to cope with them, has become one of the main purposes to achieve in the correct development of the contemporary city. The floods of the rivers being a recurrent impact in fluvial riversides and their urban banks are an opportune scenario for the study of urban-resilient behavior. "Road to Resilience" analyzes a case study of the hydrological system of the Ebro River as it flows through the city of Zaragoza, noting actions that exemplify anticipation, absorption or adaptation strategies for the city in response to floods through the Articulation of the urban and fluvial systems, the management of the urban riverbank through Planning, and the implementation of new forms of Protection, derived from a logic of defense from flooding.
\end{abstract}

\section{Keywords}

Urban resilience; Floods; Urban Bank; Zaragoza. 


\section{La necesidad de protección en la ribera urbana contemporánea}

Razones geográficas de posición (de defensa, comunicaciones o comercio) y la presencia de condiciones para el desarrollo de la vida (la fertilidad de la tierra, la disponibilidad de agua, adecuadas condiciones climáticas) han sido criterios básicos para la selección del lugar para la fundación de ciudades junto a ríos. Estas lógicas de emplazamiento han considerado la autoprotección como un requisito fundamental de carácter preventivo.

Las primeras civilizaciones de la historia, llamadas "civilizaciones fluviales", destinaban los espacios más próximos al cauce del río, la ribera fluvial, a la actividad agrícola, dedicando el suelo que no se podía inundar a la construcción de viviendas, edificaciones y espacios de comunidad. Las implantaciones originales escogieron terrenos adyacentes a sistemas naturales de agua, y se establecieron sabiendo protegerse de estos, asentándose a la distancia adecuada. En este sentido, las ciudades se establecían próximas pero nunca lindantes al curso del agua en el espacio de las avenidas. Es en la transición de la ciudad agrícola a la ciudad industrial cuando los asentamientos se hacen más complejos y desarrollan una ocupación territorial que ignora las originales lógicas de autodefensa, se ocupan las riberas de los ríos y se exponen, en consecuencia, a incidentes de inundación.

En el proceso de crecimiento y transformación urbana hacia las metrópolis o megalópolis contemporáneas, se producen nuevas situaciones de vulnerabilidad y se plantea la autoprotección como una necesidad urgente e ineludible. La ciudad contemporánea también tiene el reto de vivir las consecuencias del cambio climático, producidas como un efecto precisamente derivado del fenómeno de la urbanización, la industrialización y el desarrollo de las ciudades. Con el cambio climático, además, ha aumentado la probabilidad de episodios de crecida e inundaciones tanto en cantidad como en intensidad. Esto implica áreas de descarga fluvial extraordinaria más amplias y para la urbanización mayor espacio en riesgo y menor espacio potencialmente urbanizable. 


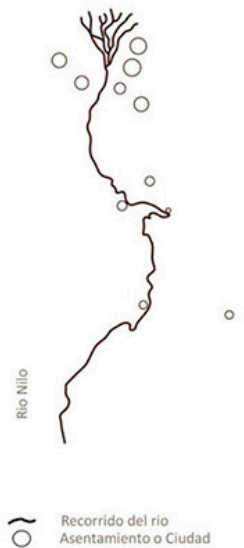

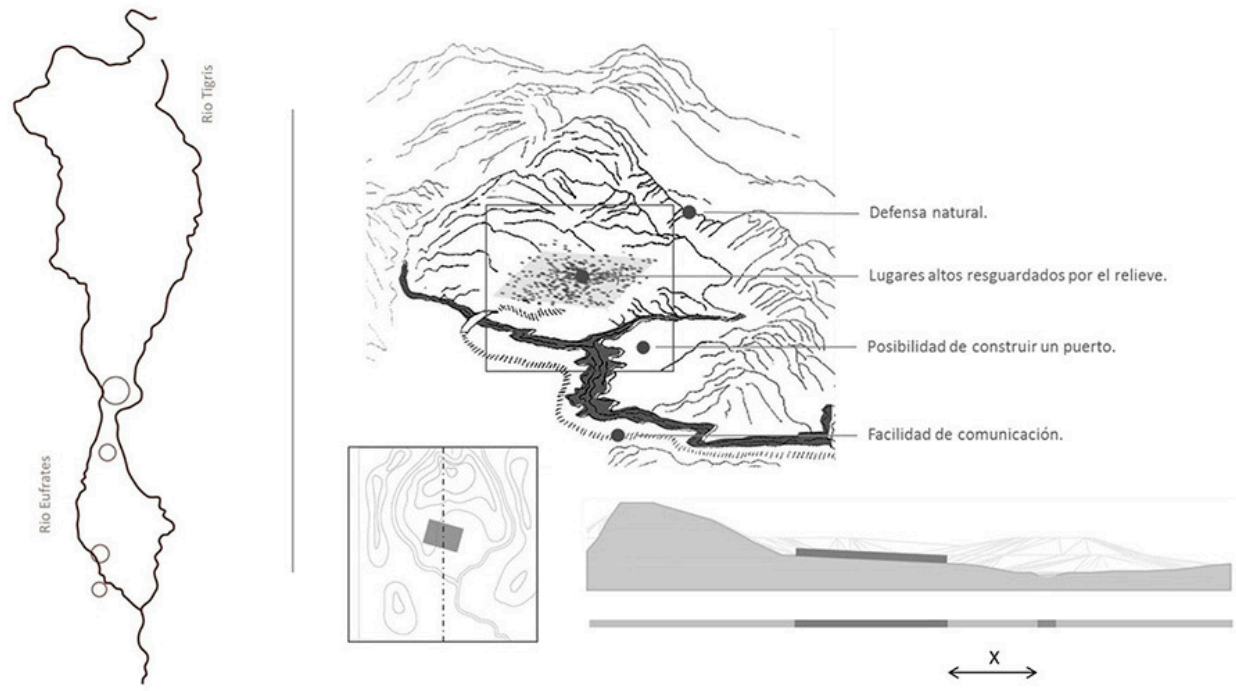

Figura 1. Características del lugar de emplazamiento de las ciudades antiguas. Fuente: elaboración propia

Las inundaciones son episodios repetitivos en las áreas urbanas situadas dentro del espacio fluvial, porque son parte del proceso que rige el sistema hidrológico. La atención a las inundaciones, consecuencia del proceso hidrológico y del cambio climático, supone una desventaja territorial destacable para la ocupación, el aprovechamiento y el uso del suelo.

El riesgo que representa la situación ambiental sumado a las propias dinámicas transformadoras de la ciudad hace relevante un enfoque de análisis urbanístico que considere de manera más integral la ocupación del territorio y la arquitectura de lo urbano, tomando en cuenta el concepto de resiliencia, que parecía presente en las lógicas usadas por asentamientos primitivos. 
Las oportunidades que ofrecían las riadas para la sociedad agrícola en el pasado amenazan actualmente la ciudad posindustrial. Por eso existe una diferencia entre la autoprotección como requisito previo en la selección del lugar de emplazamiento y la autoprotección como necesidad para responder a una problemática sobrevenida. La ciudad agrícola tenía claro que para desarrollarse debía elegir un lugar seguro y estratégicamente ubicado. La ciudad posindustrial, ya emplazada, requiere mantener su desarrollo y crecimiento. En una ciudad agraria la cuestión de la seguridad era preventiva (alejarse del riesgo). En la ciudad contemporánea, la actitud es reactiva (afrontar el riesgo).

Atendiendo a que la mayoría de las ciudades en el planeta se encuentran en contacto con cursos de agua y que vivimos en ciudades fluviales, la discusión sobre la reducción del riesgo de inundación, atención al cambio climático y la generación de capacidad urbana de respuesta o recuperación se ha convertido en tema relevante de la cuestión urbana, introduciéndose el concepto de 'resiliencia urbana'. La resiliencia inicialmente se define como la capacidad de los materiales elásticos de deformarse y volver a la posición inicial. Para entender el concepto desde la óptica disciplinar, aprovecharemos tres miradas que desde el urbanismo nos ayuden a evaluar actuaciones con vocación resiliente (deformarse y volver al estado inicial) en la ribera urbana: una es la ciudad holística; otra, la ecología urbana y por último, la resiliencia socioecológica urbana.

Los apuntes y reflexiones que introducen la 'resiliencia' en la disciplina urbanística se registran con mayor frecuencia a partir de la primera década del siglo XXI. Sin embargo, la idea de la ciudad holística de Christopher AleXANDer (1965) ya planteaba una visión del diseño urbano que contemplara el funcionamiento integral de la ciudad en relación con el medio. Es decir, la idea de una ciudad fortalecida que incluya todos sus componentes vinculados tanto con el sistema natural como con el artificial.

En 1972, la ecología urbana definió las ciudades como ecosistemas, proponiendo pensar y gestionar la ciudad de manera sistémica junto al contexto natural y el soporte geográfico. En esa época aparecieron términos como sostenibilidad ambiental, homeostasis urbana o autorregulación. Para la ecología, el proceso de homeostasis comprende dos fenómenos: el primero, la resistencia de un cuerpo que mantiene sus propiedades a pesar de ser impactado por un fenómeno externo. El segundo, la resiliencia, según el cual es posible adaptarse al impacto y volver al estado original después de la alteración sufrida. En las últimas décadas 
1. Como se podría ver en el marco de la resiliencia urbana "City Resilience Framework" definido por la Rockefeller Fundation y la consultora global de ingeniería y urbanismo ARUP. del siglo XX, las teorías sobre resiliencia urbana empezaron a abarcar distintos aspectos relativos a la ciudad. Inicialmente se orientaron hacia la economía y la gestión urbana, como las aproximaciones de Mario Polèse (2010) o Ricardo Méndez Gutiérrez del Valle (2012). En los últimos años viraron hacia la salud ambiental, el bienestar de las personas, la sociedad y el lugar, la economía, la gobernabilidad y el conocimiento ${ }^{1}$.

En 2015, los investigadores Maritza Duque y Carlos Montes (2015) realizan un análisis que incide en la compresión y aplicación del concepto resiliencia en la disciplina urbanística. Identifican dos modelos de gestión, uno de ellos es el llamado "Dominio-Control", implementado mediante la 'Resiliencia Ingeniera' (HoLling, 1996), el cual actúa para mantener lo que considera identidad permanente del sistema bajo condiciones mínimas de complejidad e incertidumbre, pretendiendo controlar lo inesperado mediante soluciones tecnológicas y haciendo "retroceder" el sistema. El segundo modelo es el de "Cambio-Adaptación", que entiende las dinámicas sistémicas como no predecibles, es decir, que existen diferentes estados alternativos a los que retroceder: "El sistema absorbe antes de que cambie su estructura y funcionamiento al pasar a otro estado alternativo". Este último modelo materializa una nueva definición de resiliencia, la "Resiliencia Socioecológica Urbana", que busca gestionar en lugar de eliminar un determinado régimen de perturbaciones. Se trata de "pasar de las ciudades diseñadas para evitar, a las ciudades que aprenden a vivir con alteraciones de baja y media intensidad" (Duque y Montes, 2015: 11-15).

La ciudad organizada bajo un pensamiento holístico, que ha superado el paradigma de la falsa dualidad entre lo natural y lo artificial (DuQue y MonTEs, 2015), acude a los mecanismos que armonizan los sistemas río y ciudad, en la inevitable relación que comparten. Ríos y ciudad que aumentarán su interacción — como sabemos- a consecuencia del cambio climático, y que nos hacen pensar en la ribera y su ordenamiento fluvial-urbanístico, como entendemos que acertadamente apunta José María Ureña Francés (2012), en su conferencia "La Ordenación de los Espacios Fluviales en las Ciudades", cuando señala lo que él considera algunas de las características del territorio fluvial que la ribera urbana necesitaría acompasar por medio de la ordenación espacial:

Cambio. Los ríos están en permanente cambio y su morfología se ha generado especialmente para adaptarse a dichos cambios. En consecuencia, la ordenación de las áreas fluviales debe permitir un cierto margen para que ellos se produzcan. 
Diversidad. Los ríos no son uniformes. En consecuencia, la ordenación de un área fluvial no puede hacerse estableciendo un modelo uniforme.

Frontera. Las áreas fluviales están compuestas siempre por la unión entre lo acuático y lo terrestre. En consecuencia, la ordenación de las áreas fluviales debe considerar inseparables ambos ámbitos y la relación entre ellos.

Sistema. Un tramo de un río no puede aislarse del resto. La ordenación de un tramo del sistema fluvial influye en los demás, y debe realizarse con un enfoque de conjunto.

Territorio. Los ríos no están limitados a su cauce, sino que su territorio está formado por un espacio más amplio. En consecuencia, la ordenación de los ríos debe realizarse conjuntamente con la de su llanura de inundación.

En la combinación o aplicación práctica de criterios de ordenación fluvial, ordenación urbanística y la idea del modelo de gestión "Cambio-Adaptación”, se perfila un instrumento de transformación y adecuación de la ribera urbana para su protección, como base de orientación para las intervenciones de manejo de inundaciones, que permita retomar una relación eficiente entre ambos sistemas. Es necesario hacer la salvedad de que la resiliencia es un concepto abarcador que como un objetivo del urbanismo debe tomar en cuenta otros ámbitos urbanos, además de los analizados en este artículo, como ya habíamos referido: la economía, la consumición y producción de energía, el aprovechamiento de los recursos, la gestión, gobernabilidad y muchos más.

Nuestra preocupación se concentra en el ámbito particular de estudio territorial y geográfico de la ribera urbana y en cómo, para que sea realmente urbana, reúne o hacemos que reúna ciertas condiciones que permiten que alcance un comportamiento resiliente. Ante el riesgo y las problemáticas que representan las inundaciones en el contexto urbano nos preguntamos ¿de qué manera puede actuar la urbanística para contribuir a la generación de una ciudad más resiliente?; ¿mediante qué instrumentos o recursos se puede lograr?

La problemática demanda la selección de una ribera urbana que haya ejemplificado estrategias de autoprotección, anticipación, absorción, adaptación o recuperación ante las inundaciones por crecidas a lo largo de los años para analizar, escogiéndose la ciudad de Zaragoza y los espacios fluviales urbanizados alrededor del río Ebro, aunque podría haber sido otra. 


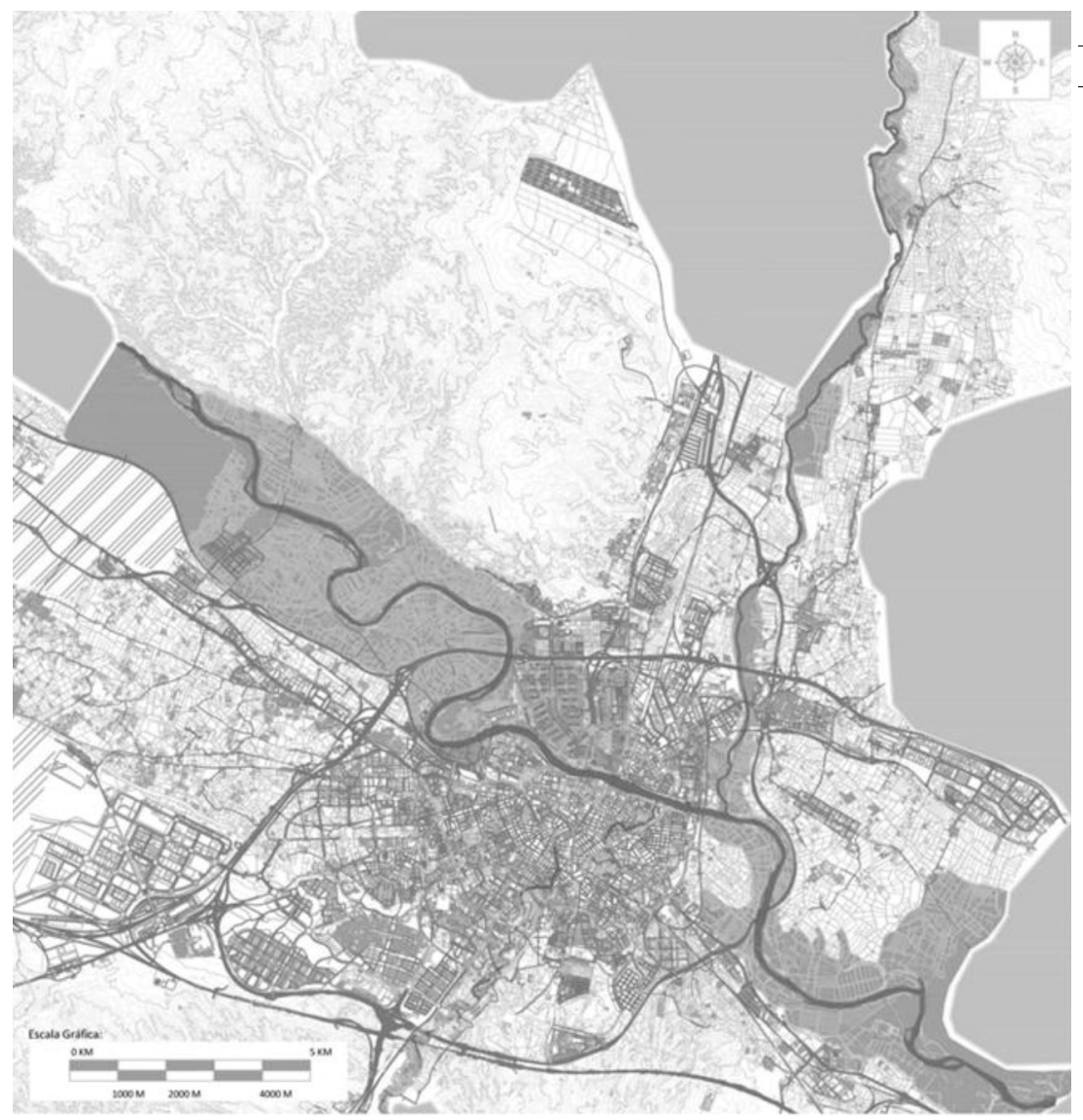

Figura 2. Zaragoza, la geografía inundable. Fuente: elaboración propia
El caso de estudio: el Ebro en Zaragoza (España)

Zaragoza es una ciudad implantada en el valle medio de la cuenca del río Ebro, en la confluencia con los ríos Gállego y Huerva. La ciudad al formar parte de este sistema hidrológico se ve expuesta periódicamente a crecidas fluviales. En la posición geográfica en que se encuentra la ciudad, el río Ebro es capaz de alcanzar un caudal de $2000 \mathrm{~m}^{3} / \mathrm{s}$ cada dos años y hasta 4300 $\mathrm{m}^{3} / \mathrm{s}$ en crecidas extraordinarias, de $1000 \mathrm{~m}^{3} / \mathrm{s}$ en el Gállego y de $50 \mathrm{~m}^{3} / \mathrm{s}$ en el Huerva. Los registros de inundaciones a lo largo de la historia para Zaragoza muestran que desde el año 1600 a 2015 se produjeron quince episodios importantes de inundación.

Se considera ribera urbana el territorio que abarca la mancha de inundaciones periódicas de retorno cada cien años, contemplando las llanuras de inundación de los ríos Ebro, Gállego y Huerva. La fundación de Zaragoza (500 a. C.) en el valle medio del Ebro se realizó en la parte más elevada, en la margen sur, muy cerca del río y considerablemente alejada del río Huerva, como estrategia para servir de puente entre los demás pueblos iberios (GuINEA, 2012). La posición geográfica de 
Zaragoza, auto-protegida respecto del río y su localización estratégica dentro de la península ibérica, fue aprovechada desde la época del Imperio Romano. La colonia Cesaraugusta (Zaragoza, 14 a. C.) geometrizó su trama para la higiene urbana: su trazado ortogonal se orientaba cardo-cardines para desaguar en el Ebro, decumano-decumani para desaguar en el Huelva, mientras que el agua potable provenía del Gállego por medio de un acueducto, única razón por la que la ciudad plantea la ocupación "del otro lado", que en principio se dedicaba solo a la agricultura. Los ríos estaban al servicio de la ciudad y las riberas inundables no eran ocupadas (GUINEA, 2012).

Los principales cambios y transformaciones del desarrollo urbano de Zaragoza datan de la época musulmana (711 a. C.) y de la instauración del reino de Aragón (1118 d. C.) en la Edad Media, hasta la Edad Moderna en el siglo XVII. Estas últimas transformaciones poco tuvieron que ver con la presencia de los cauces fluviales en el territorio o la presión de sus dinámicas internas sobre ella. En el año 1600, Zaragoza creció y ocupó la ribera de la margen norte, que era inundable.

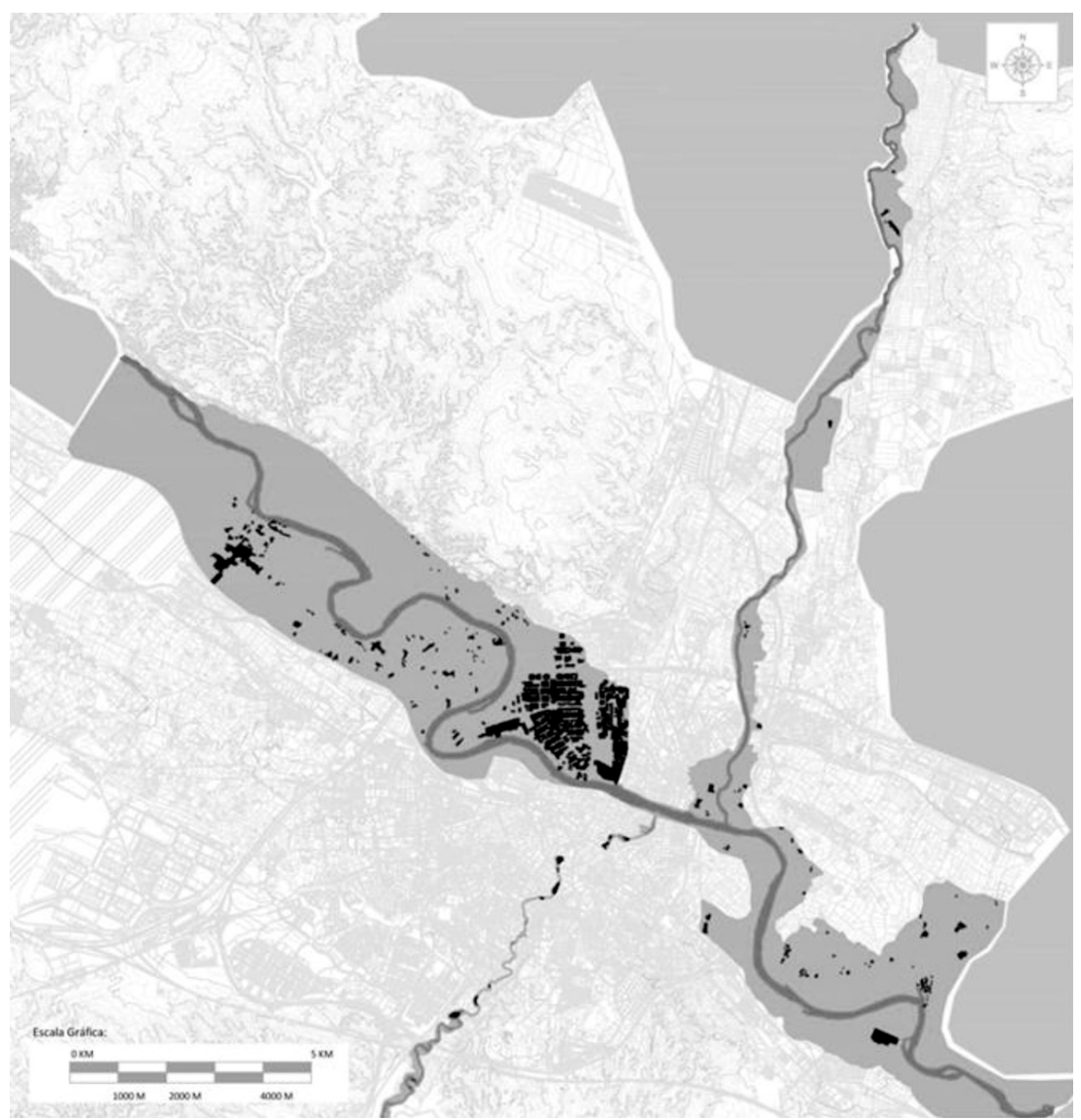

Figura 3. "Ribera urbana de Zaragoza".

Fuente: elaboración propia Desde entonces ensaya maneras de defenderse de las avenidas. 
2. Estos son: "Plan General de Ordenamiento Urbano de Zaragoza" (1986),

"Concurso Nacional de

Idea para la ordenación de las riberas" (1989),

"Anteproyecto de espacios públicos de la ribera urbana

del río Ebro" (2001), "Plan

General de Ordenamiento Urbano de Zaragoza"

(2002), "Plan Director de la

Expo Zaragoza 2008 y su

Plan de Acompañamiento" (2004), "Proyectos del

Plan de Riberas del Ebro" (2005) y el "Plan Director de Recuperación del río Huerva" (2009).
Durante mucho tiempo, ríos y riberas son vistos como lugares conflictivos, y se actúa duramente sobre el espacio de las crecidas, encauzando el río. Las primeras soluciones aplicadas a los efectos de las crecidas tenían un carácter reactivo, que en la práctica se basaba en reconstruir, reponer y sustituir construcciones, infraestructuras u otros bienes dañados. A inicios del siglo XX fueron empleadas, de acuerdo con el Ayuntamiento dE Zaragoza (2011), las primeras medidas preventivas de aviso por medio de medios tecnológicos de detección de crecidas, como la instalación de estaciones de aforo en la cuenca del río aguas arriba y un Sistema Automático de Información Hidrográfica (SAIH), que no tenían ninguna implicación con el urbanismo que se desarrollaba. Después de la riada de 1961, se construyeron en la ribera del Ebro en Zaragoza diques longitudinales de tierra en la margen izquierda de aproximadamente 1,6 km de largo y alturas entre 2,5 y $4 \mathrm{~m}$. Estas medidas hidráulicas de ingeniería tampoco se llevaron a cabo con algún fundamento urbanístico, y en 1966 debieron ser reconstruidas tras el impacto de las crecidas ese año, en que también se inició un proceso de estabilización del cauce del Ebro de colocación de diques y escolleras.

A partir de 2008, la ciudad, en el contexto de la Exposición Internacional del Agua, invierte en su desarrollo con la visión de recuperar e integrar las riberas, introduciendo un cambio vertiginoso en el urbanismo que se había hecho hasta entonces, prestando atención a los espacios fluviales y al río Ebro para poder ser utilizado como elemento urbano. Las intervenciones en la ribera pasan de ser puntuales, duras, y puramente materia de ingeniería, a complementarse en un conjunto de actuaciones de carácter blando, respetuoso, desde un enfoque holístico, multidisciplinar, que incluye aspectos de paisaje, de recuperación y conservación del medio físico.

La última transformación de la ribera urbana de Zaragoza, a través de instrumentos de planificación y otras iniciativas urbanísticas ${ }^{2}$ ha llevado a cabo actuaciones que se pueden agrupar en tres niveles: articulación, relacionadas a la estructuración de un único sistema en el que se integran las riberas urbanas y el resto de la ciudad; ordenación, que sirven para dotar de funcionalidad y aprovechamiento de los espacios fluviales con usos y actividades urbanas, y por último, protección, actuaciones materiales blandas e "inteligentes" de defensa de los espacios fluviales-urbanos, que finalmente aportan espacios fluviales-urbanos resilientes desde las lógicas de anticipación, adaptación, absorción y recuperación. Estas estrategias generalizables se convierten en prototipos de intervención en casuísticas diversas. 


\section{NIVEL DE INTERVENCIÓN 1: ARTICULACIÓN}

Este nivel de intervención hace de las realidades presentes en el territorio, lo natural y lo urbano, un conjunto estructurado, articulando los tres ríos (Ebro, Gállego y Huerva) con la ciudad (Zaragoza). Su objetivo se resume en unir, relacionar y hacer posible el movimiento, a través de la creación de una red de espacios abiertos continuos como infraestructura verde, en los que se incluye el sistema fluvial que comprende las márgenes de los tres ríos. Se consigue conectar y al mismo tiempo integrar y estructurar el sistema, haciendo una aportación en cuanto a absorción y adaptación como consecuencia de algunas de crecidas fluviales. Se hace a través de actuaciones como la red de espacios abiertos o "Anillo Verde", las infraestructuras de vinculación y conexión, el acceso a través de una relación física con las redes de transporte público y la diversificación de medios para moverse dentro de los espacios fluviales-urbanos.

\section{"Anillo Verde"}

Para articular todo el sistema, de acuerdo con el "Plan de Acompañamiento de la Expo Zaragoza 2008”, la ciudad integró sus espacios libres, que en su mayoría son adyacentes a los cursos de agua, proyectándose el "Anillo Verde de Zaragoza" como sistema vertebrador de la ciudad y elemento de delimitación del crecimiento urbano. Hoy el anillo verde comprende el río Ebro y el Canal Imperial de Aragón enlazados por del corredor verde Oliver-Valdefierro al oeste de la ciudad y una segunda vía en el límite este. Esta delimitación se conoce como el Anillo Sur. El Anillo Norte comprende el eje del Ebro y el Gállego, que ha quedado abierto hasta el momento. El anillo completo aparece proyectado en el Plan Director de Recuperación del río Huerva de 2009.

\section{Infraestructuras de vinculación y conexión}

Articular se define como la acción de unir, relacionar y hacer posible el movimiento. Esto se puede conseguir conectando y dando continuidad, es decir, vinculando núcleos urbanos mediante infraestructuras, uniendo espacios fluviales, el territorio metropolitano y la ciudad mediante el transporte público, lo que ayuda a los espacios de ribera a ser accesibles o a enlazar los municipios de la metrópolis. 


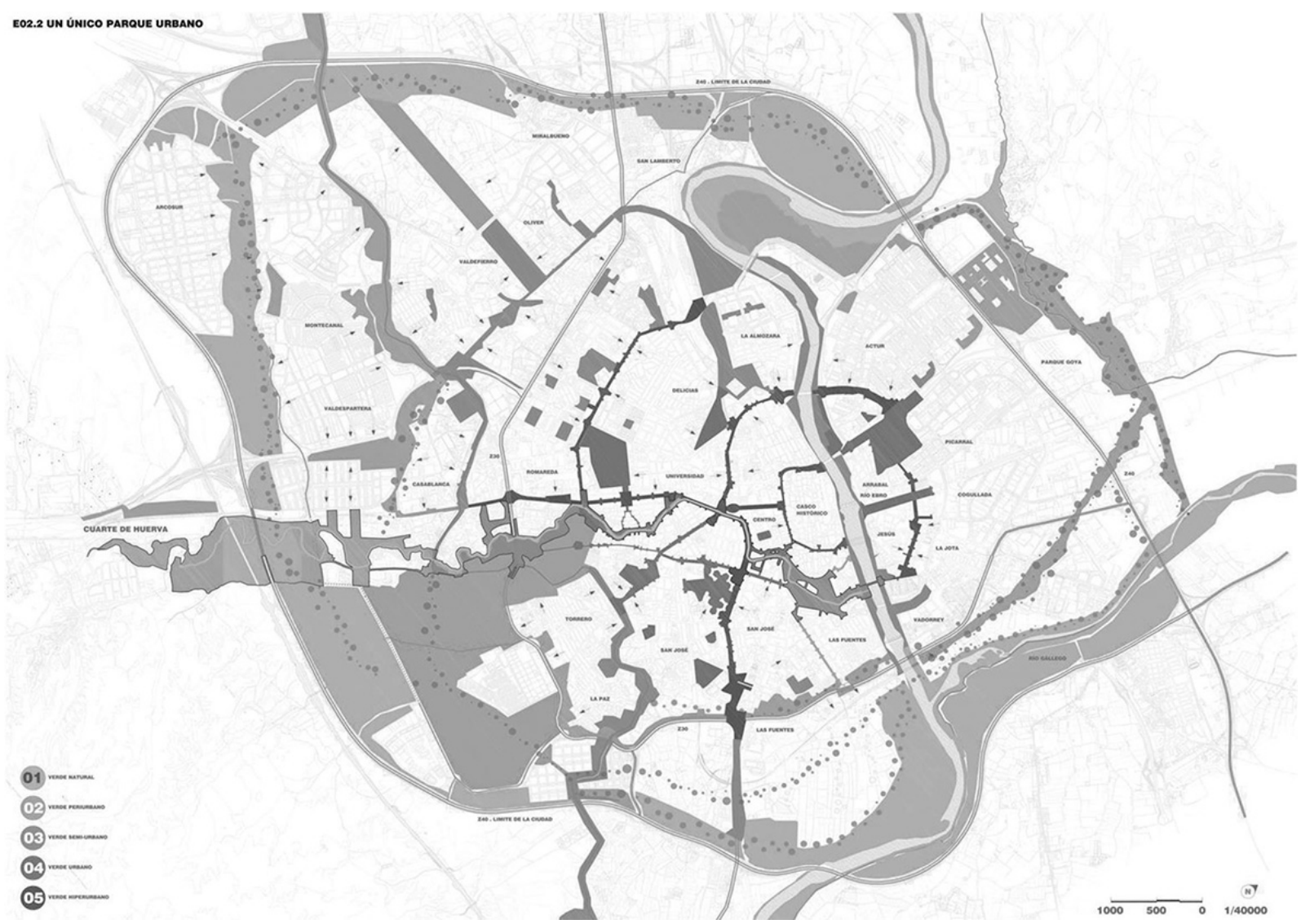

Figura 4. Proyección del Anillo Verde de Zaragoza. Fuente: Plan Director del río Huerva (2009). Ayuntamiento de Zaragoza, España 
En el caso de Zaragoza, para que la estrategia del "Anillo Verde" funcione como tal, ha de conectar los espacios verdes y abiertos de toda la ciudad (centro-periferia-urbano-metropolitano). En la actualidad, las vías que siguen el recorrido de los ríos, delimitando de alguna forma la ribera urbana, se conectan a la infraestructura viaria de todo el territorio, lo que permite la comunicación de estos elementos lineales con el resto de la estructura, y viceversa. Esta continuidad o enlace es más que una forma de vertebración del tejido cuando permite el movimiento.

\section{Acceso y movimiento en los espacios fluviales-urbanos}

Otra manera de generar esta continuidad consiste en la diversificación de los medios de acceso a través de la priorización del transporte público y continuidad peatonal (CARCAÑo, 2015), ya no solo conectados a la trama urbana, sino también haciendo posible la relación de todo el sistema fluvial. En el caso de Zaragoza, el servicio de transporte público que se diversifica en líneas de autobuses, tranvía y trenes que circulan sobre una trama viaria que se conecta con los espacios fluviales y recorren o se acercan a la ribera urbana. La continuidad de los espacios abiertos en la ribera urbana se realiza por medio de pasarelas. Aunque el "Proyecto de Márgenes y Riberas Urbanas del río Ebro en Zaragoza" dividía la ribera en tramos urbanos, es unida por estas pasarelas peatonales con carril bici, que además permiten el acceso de vehículos de mantenimiento o de emergencia.

\section{La articulación capaz de generar resiliencia}

Las actuaciones de "articulación" contribuyen a la absorción del impacto de las crecidas. Normalmente cuando los espacios fluviales-urbanos están inundados, se asume que estarán inactivos y que la parte de la ciudad que allí se encuentra podría perder lugares que habitualmente usa. Sin embargo, el funcionamiento de una estructura bien conectada hará que la parte no inundada de la ciudad absorba el flujo de los ciudadanos que habitan la ribera urbana. Las áreas que quedan fuera de la ribera urbana tampoco pierden espacios funcionales a causa de una inundación, porque la ciudad ha diseñado y reservado espacios verdes fuera del territorio fluvial para satisfacer estas necesidades (figura 5). 


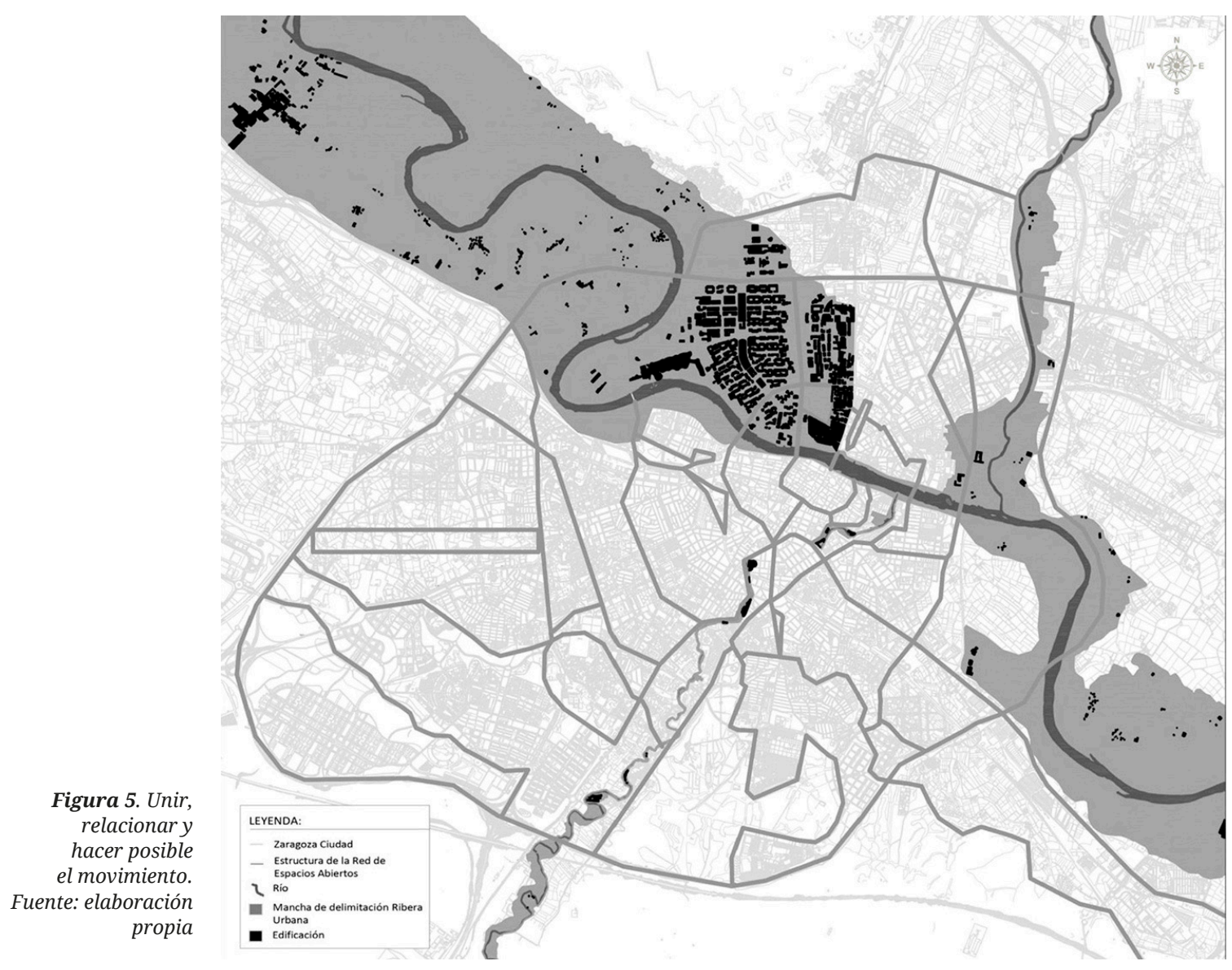




\section{NIVEL DE INTERVENCIÓN 2: ORDENACIÓN}

Los espacios fluviales son lugares condicionados por las dinámicas del río. No obstante, de igual manera son espacios útiles que se prestan a diversas utilizaciones. Las actividades asignadas a la ribera urbana y la configuración espacial de los proyectos y elementos cerrados que se construyen en ella forman parte de las actuaciones que se agrupan en un segundo nivel de intervención, el de "ordenación”, relativo a la disposición de usos y actividades, normas de ordenamiento y configuración espacial de los proyectos de barrio en la ribera urbana.

Estas actuaciones persiguen la utilidad y funcionalidad del espacio en un contexto diverso y condicionado, además de configurar espacios en los que la inundación ya no sea un problema. Entre ellas están la asignación de usos distintos de los de conservación, la creación de mayor proporción de vacíos funcionales que de llenos construidos o edificaciones, la configuración del proyecto residencial a partir del bloque aislado.

\section{Asignación de usos y diversificación de actividades}

En las riberas de los ríos Ebro, Gállego y Huerva aparecen usos de equipamiento ciudadano, espacios naturales de interés, verde urbano, instalaciones militares, uso residencial, administrativo e industrial. Predominan los dedicados al verde, en primer lugar; después, los dedicados a dotación. El uso residencial es el que aparece en menor proporción, y en él se admiten actividades como el verde urbano, el comercio, edificaciones para dotaciones, entre otras.

Los usos de las riberas se ordenan dependiendo del riesgo de inundación: en las zonas inundables cada uno a cinco años se mantiene el estado natural del suelo sin construcción. A partir de los cinco años hasta los veinticinco años se permiten usos: agricultura, vías de circulación e instalaciones deportivas (Ayuntamiento de Zaragoza, 1999) (figura 6).

\section{Creación de mayor proporción de vacíos funcionales}

En la configuración de la ribera urbana zaragozana el vacío es la norma, entendiendo como vacío el espacio libre de edificación o espacio abierto dentro de la trama. Podemos 
Figura 6

Ordenación del territorio fluvial-

urbano. Fuente: elaboración propia

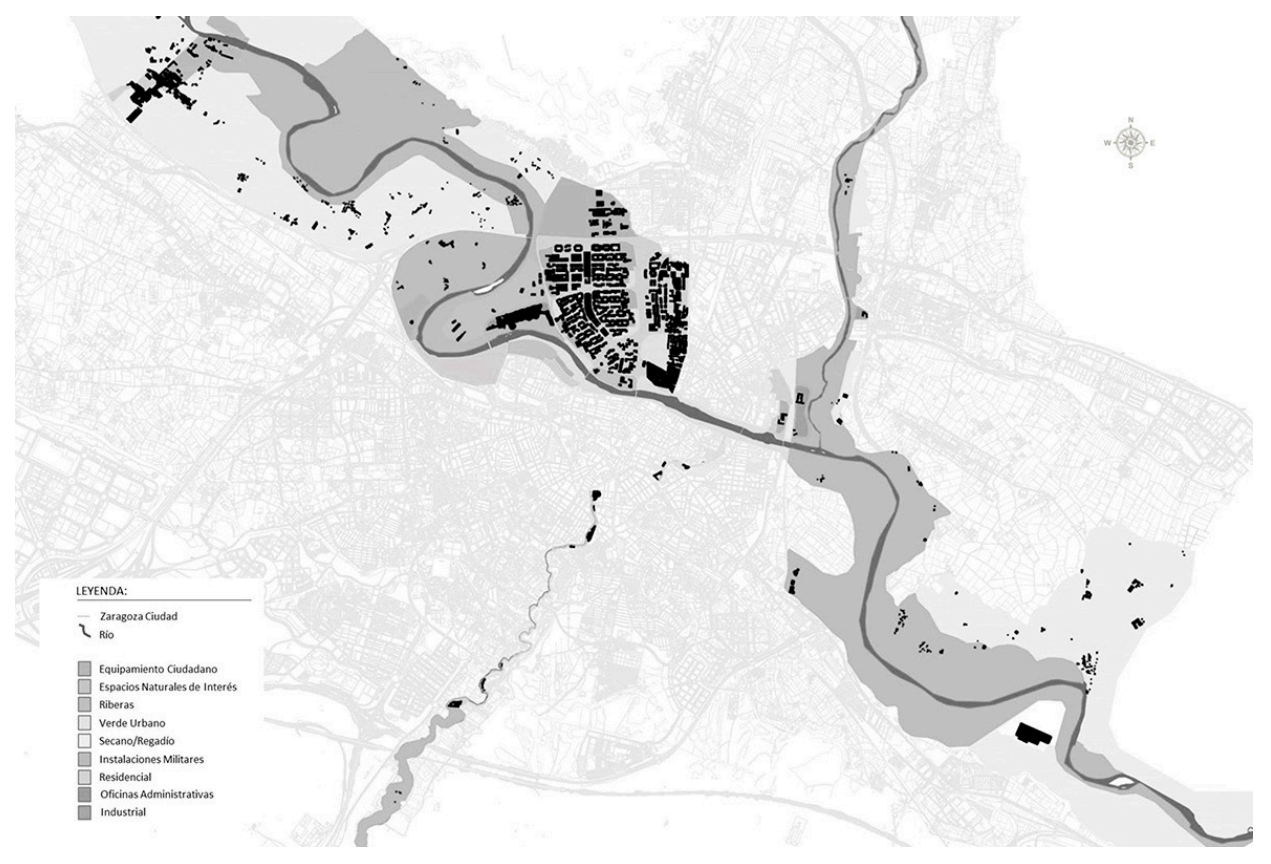

ver que la ribera es un gran vacío donde los proyectos de edificación, ya sean de equipamiento, vivienda u otros, van sumando otros espacios abiertos como parte integral de su lógica proyectual o arquitectónica. Una proporción del $75 \%$ al 85 \% del territorio está destinada a vacíos funcionales, espacios sin construcción pero con asignación de uso y actividad.

La proporción de superficie sin edificar es equivalente a la superficie construida en la zona de inundación. De aproximadamente $56,21 \mathrm{~km}^{2}$ de superficie que posee toda la ribera urbana, unos $13,73 \mathrm{~km}^{2}$ representan el suelo edificado, es decir, casi $1 / 4$ parte del territorio fluvial en el que viven alrededor de 84,368 personas. 


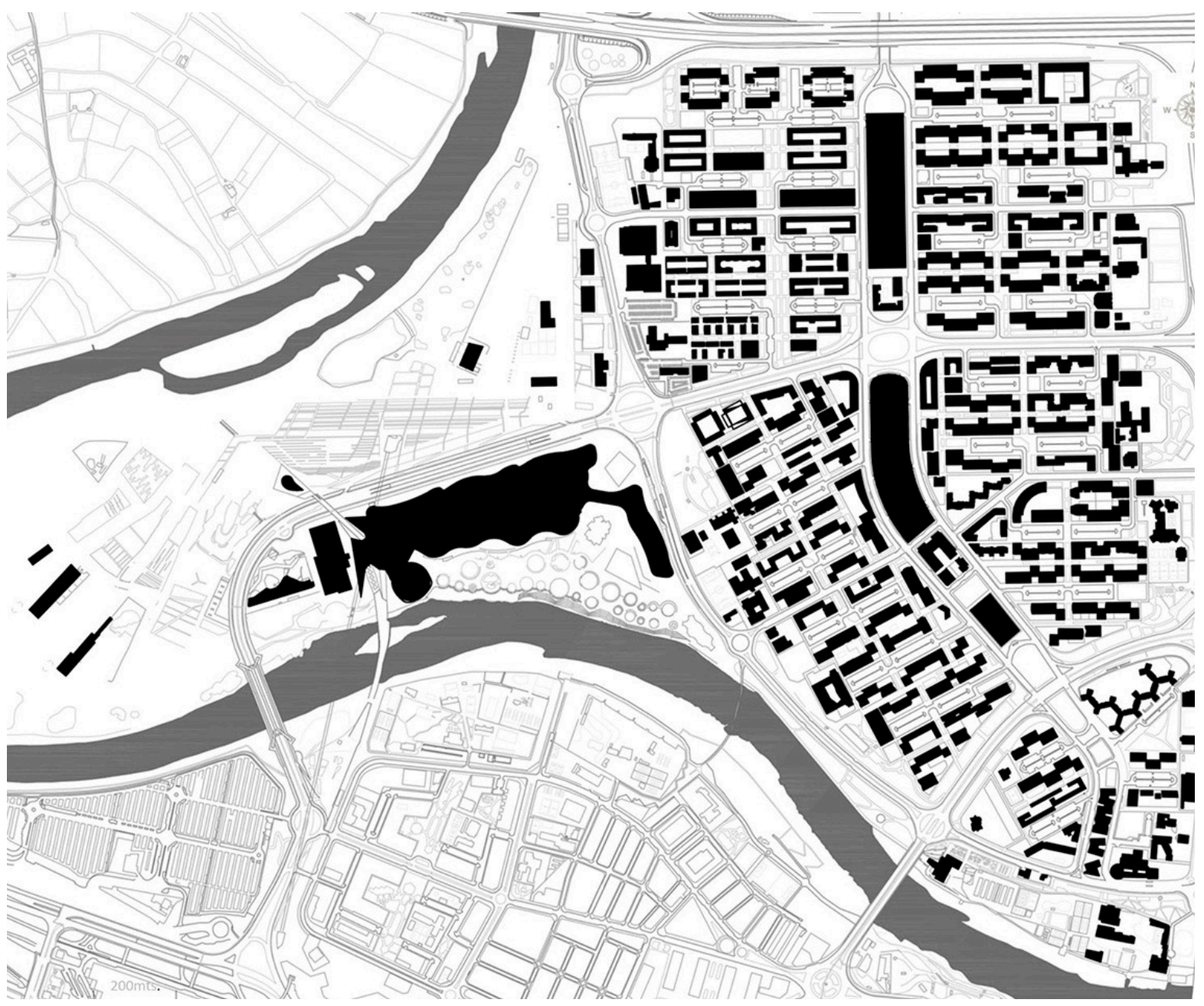

Figura 7. Configuración espacial del barrio Actur dentro de la ribera urbana de Zaragoza. Fuente: elaboración propia 


\section{Configuración del proyecto residencial desde la lógica de mayor proporción de vacío funcional}

Llama la atención la relación de llenos y vacíos, y la singular estructura del tejido urbano del barrio Actur, distinta de la encontrada en la zona consolidada u otros barrios dentro de la zona de ribera. Es el proyecto urbano de mayor magnitud comprendido por completo dentro de la zona inundable. El conjunto sigue el modelo de clúster, basado en una arteria central con dos grandes vías que delimitan terrenos destinados a equipamientos, cruzadas por arterias transversales a las que se adosan las "unidades de barrio" separadas por vías de distintos niveles y pequeñas explanadas o jardines. La ocupación de la parcela es baja y la tipología edificatoria el bloque aislado sin ocupar toda la manzana.

Algunas ventajas de la construcción en clúster dentro de la zona inundable es la cantidad de espacio libre de edificación que ayuda al desagüe y absorción del agua de inundación a través del suelo; la dimensión corta de fachada permite que el agua se direccione más fácilmente según su movimiento y se agilice su evacuación. El tamaño de las vías, los distintos niveles según el uso que tienen facilitan la circulación y/o evacuación de personas en estos lugares de ser necesario.

\section{La ordenación capaz de generar resiliencia}

La ordenación de riberas urbanas se fundamenta en un conjunto de medidas orientadas a la especificidad del lugar (terreno inundable), resultado del estudio de las crecidas, pero que hay que planificar, proyectar y adaptar. La ciudad contemporánea no se puede permitir el abandono o desaprovechamiento de este territorio, aunque sea un lugar "difícil". Habitar correctamente el espacio de ribera permite su ocupación sin que la crecida sea un problema para la ciudad. Se arriesgan menos usos y menos edificación, se conserva la productividad del suelo, el medio natural, y se pueden diversificar las actividades. Ordenar con atención a las dinámicas fluviales ayuda a marcar pautas de proyectación que se adapten al comportamiento del río, de manera que la configuración acepte la entrada y salida del agua. Medidas que no impiden la inundación ni que la ciudad o los espacios fluviales continúen funcionando. 


\section{NIVEL DE INTERVENCIÓN 3: PROTECCIÓN}

Otro aspecto considerado en el tratamiento y transformación de la ribera urbana de Zaragoza agrupa los mecanismos de mitigación de impacto de las crecidas, actuaciones de carácter físico o material y obras de ingeniería para la protección del espacio geográfico expuesto, así como de la ciudad misma. Este nivel, relativo a las actuaciones materiales hidráulicas, infraestructuras y obras de defensa, reúne tres grupos de actuaciones: defensa y tratamiento topográfico, de desagüe y manejo del agua en caso de inundación y medidas de estabilización del caudal.

\section{Defensa y tratamiento topográfico}

Las obras hidráulicas de defensa buscan canalizar y conducir el agua, mientras que las de tratamiento topográfico protegen las pequeñas laderas formadas por los cambios en el nivel topográfico. De este modo, se reduce el impacto o gravedad de las crecidas. Sin impedir la inundación, manejan la fuerza del movimiento del agua cuidando el tejido urbano. Por ejemplo, la plaza de la Exposición Internacional ${ }^{3}$ se construye sobre un dique invisible o talud vegetado/escalonado que permite el acceso peatonal y en bicicleta por medio de escaleras y rampas. La protección del talud a lo largo del tramo se realiza a través de medios blandos, como escolleras con vegetación y bloques de hormigón entrelazados. Otro elemento de defensa importante en el manejo de las inundaciones es el muro de contención que está localizado en la margen izquierda del Ebro, a la altura del barrio Actur. El muro fue tratado para funcionar como elemento urbano mejorando su aspecto, protegiéndolo y diseñándolo de manera que funcione como pasarela elevada o mirador.

Para la accesibilidad y el movimiento dentro de los espacios libres en la ribera, se construyeron paseos con el fin de orientar el uso de determinadas zonas dependiendo de la situación hidráulica: el paseo inferior se puede usar cuando el nivel del agua alcanza el caudal de $250 \mathrm{~m}^{3} / \mathrm{s}$ en el Ebro; el paseo intermedio, a nivel de avenidas ordinarias, cuando el caudal alcanza los $2000 \mathrm{~m}^{3} / \mathrm{s}$ y el paseo superior, el único que se puede usar en avenida extraordinaria.
3. La plaza de la Exposición Internacional es una plataforma entre el río Ebro y el recinto de la Expo Zaragoza 2008. 


\section{Desagüe, canalización y manejo del agua}

Se sitúan en este grupo las obras hidráulicas utilizadas para conducir el agua y desocupar el territorio inundable fácilmente, de la manera más natural y a la mayor rapidez posible, como represas para contener el agua por un corto período. Así ocurre en el meandro de Ranillas, a partir de la construcción del Parque del Agua que canaliza las aguas para inducir una reducción de su nivel y que consigue en algunas ocasiones que con el propio diseño se flexibilice la duración del fenómeno en el parque.

El parque recupera un sistema de riego del tejido agrícola que se encontraba en la zona por medio de las acequias, conformando un sistema de desagüe que estructura el meandro desde la entrada al parque por el norte hasta su llegada al Ebro. En su recorrido mejora la calidad del agua del río, al final recicla parte del agua y devuelve la otra. Toda la estructura está rodeada por un camino elevado o mota que la resguarda de las avenidas extraordinarias de $3000 \mathrm{~m}^{3} / \mathrm{s}$ en un período de retorno de veinticinco años. El parque capta, almacena y depura el agua, al mismo tiempo que recrea el espacio con ella.

\section{Estabilización del caudal de ríos}

El Azud del Ebro es una obra de ingeniería multifuncional, adaptada al programa urbano, cuya función más importante es reducir la velocidad del agua estabilizando la lámina para aprovechar la navegabilidad o mantener por un tiempo prudente un caudal recomendado. Con la estabilización del caudal disminuye la necesidad del empleo o construcción de elementos de defensa. Al mismo tiempo, esta infraestructura es nexo de unión entre las márgenes del río al funcionar con una pasarela peatonal construida sobre los elementos de apoyo de las compuertas.

\section{La protección capaz de generar resiliencia}

Adaptarse para recuperarse implica no evitar la afectación y si hay daños o consecuencias manejarlos hasta que vuelva a funcionar con normalidad. Por eso, las actuaciones materiales de protección (defensa, desagüe y estabilización) están orientadas a construirse en lugares estratégicos para resolver una situación puntual, es decir, ninguna medida de defensa es 
generalizable a toda la ribera urbana o en todo el recorrido lineal de una margen. De modo que lo que va a producir un comportamiento resiliente no es la obra hidráulica, sino cómo esta se implementa.

El principal aporte de estas actuaciones ha sido la ruptura del paradigma de contención y canalización rígida de las crecidas, que las llevó al concepto de "dejar inundar".

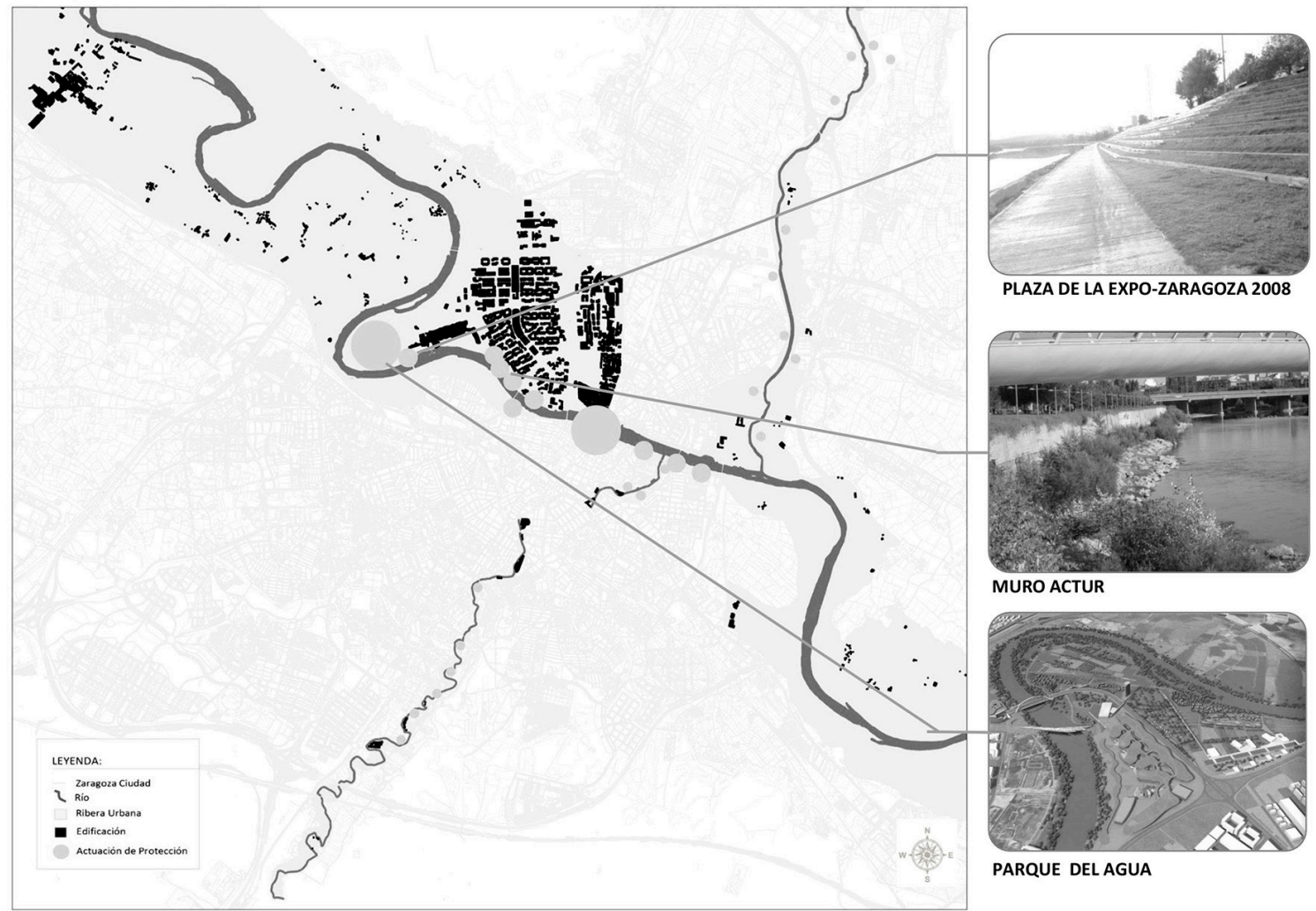

Figura 8. Protección estratégica. Fuente: elaboración propia 
En su conjunto, las acciones y estrategias analizadas muestran una sensibilización por parte de los agentes urbanísticos para interpretar y adaptarse al fenómeno de las crecidas, así como las dinámicas propias de los espacios fluviales para proteger la ciudad gestionan el proceso de modo de que existan espacios disponibles que no lleguen a inundarse, para que las avenidas ordinarias no impidan las actividades en las riberas y se logre que los espacios se adapten una vez inundada la llanura. Cada nivel de intervención expuesto y analizado tiene su interdependencia con los otros. Aunque no sean parte integral de una misma estrategia o plan, podrían llevarse a cabo en conjunto con la plena intención de generar resiliencia en el territorio. El resultado que se puede enriquecer con otras medidas, actuaciones o niveles de intervención se tendría que adaptar a la realidad río-ciudad en el que se pretenda utilizar como guía para el tratamiento y protección de una ribera urbana.

El caso del Ebro en Zaragoza es representativo de cómo se han ido estableciendo las relaciones río y ciudad a lo largo de los siglos en muchas ciudades. Primero situándose a una distancia prudencial para evitar los problemas, después levantando muros y protecciones y, finalmente, integrando las riberas al espacio urbano con recursos diversos. Las medidas son de naturaleza muy distinta, las primeras obvian el problema (durante muchos años, muchas ciudades han dado la espalda a los cursos de agua, actitud que todavía persiste en muchos lugares), después fueron defensivas (se encauzan y canalizan, se dominan las aguas) y finalmente proactivas (se proyecta con el agua), más acordes con el pensamiento contemporáneo de integración que de confrontación.

Incorporar estas y otras medidas en la reflexión sobre la ribera urbana teniendo en cuenta el relevante papel que juegan en las ciudades significa prestar atención a un componente ambiental fundamental como son los cursos de agua en las ciudades, para convertir el riesgo en oportunidad y hacer las ciudades más robustas y en consecuencia resilientes. Los cursos de agua son un elemento fundamental del proyecto urbano, y pasan de ser un problema que evitar a convertirse en la oportunidad de transformarse en un lugar central.

\section{Bibliografía}

ALEXANDER, Christopher (1971) Tres Aspectos de Matemáticas y Diseño: La Estructura del Medio Ambiente. Tusquets, Barcelona. 
AYUNTAMIENTO DE ZARAGOZA. "Plan General de Ordenamiento Urbano de Zaragoza" (1999). Ayuntamiento de Zaragoza, Zaragoza.

AYUNTAMIENTO DE ZARAGOZA. "El Ebro y sus riberas" (2000). Ayuntamiento de Zaragoza, Zaragoza.

AYUNTAMIENTO DE ZARAGOZA. "Anteproyecto de espacios públicos de la ribera urbana del río Ebro" (2001). Ayuntamiento de Zaragoza, Zaragoza.

AYUNTAMIENTO DE ZARAGOZA. "Proyecto de Márgenes y Riberas Urbanas del río Ebro. Zaragoza” (2005). Ayuntamiento de Zaragoza, Zaragoza.

AYUNTAMIENTO DE ZARAGOZA. "Plan Director de Recuperación del río Huerva” (2009). Ayuntamiento de Zaragoza, Zaragoza.

AYUNTAMIENTO DE ZARAGOZA (2011). "El Ebro desbordado. Una historia de las crecidas del río en Zaragoza”. Ayuntamiento de Zaragoza, Área de Cultura, Medio Ambiente, Participación Ciudadana y Grandes Proyectos, Agencia de Medio Ambiente y Sostenibilidad, España.

CARCAÑo Z, Carme (2015). "El río que nos lleva... al jardín de la metrópolis”. Tesina de máster. Máster de Investigación en Urbanismo. Universidad Politécnica de Cataluña, Barcelona.

GUINEA, Darío Marcos (2012). "Breve Historia del Urbanismo en Zaragoza”. Trabajo de Historia la Arquitectura. Universidad de La Coruña, Coruña.

HOLLING, C. (1996) "Engineering resilience versus ecological resilience”. En S. P. (ed.) Engineering with in ecological constraints. Pp. 31-44: National Academy Press, Washington.

MÉNDEZ, Ricardo (2012). "Ciudades y metáforas: sobre el concepto de resiliencia urbana”. En Ciudad y Territorio, Estudios Territoriales. Vol. XLIV, No. 172, España.

MONTES, Carlos; DUQUE, Maritza (2015). “Ciudades Resilientes en el Antropoceno: Mito o Realidad”. En Ciudad y Territorio, Estudios Territoriales, No. 183, Madrid.

POLÉSE, Mario (2010). "The resilient city: on the determinants of successful urban economies”. Working Paper, 2010-03. Centre-Urbanisation Culture Société. INRS. Pág. 24. University of Quebec, Montreal.

UREÑA FRANCÉS, José María (2002). "La Ordenación de los Espacios Fluviales en las Ciudades”. Ríos y Ciudades. Aportaciones para la Recuperación de los ríos y las Riberas de Zaragoza. Pp. 45-63. Diputación de Zaragoza, Zaragoza. 